\section{CVIA}

REVIEW ARTICLE

pISSN 2508-707X / eISSN 2508-7088 https://doi.org/10.22468/cvia.2018.00045 CVIA 2018;2(2):85-96

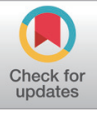

\title{
Four-Dimensional Flow Magnetic Resonance Imaging for Cardiovascular Imaging: from Basic Concept to Clinical Application
}

\author{
Hideki Ota', Satoshi Higuchi', Wenyu Sun'1, Takuya Ueda', \\ Kei Takase', Hajime Tamura² \\ 'Department of Diagnostic Radiology, Tohoku University Hospital, Sendai, Japan \\ ${ }^{2}$ Division of Medical Physics, Tohoku University Graduate School of Medicine, Sendai, Japan
}

\begin{abstract}
Magnetic resonance imaging (MRI) plays an important role in evaluation of the cardiovascular system. Two-dimensional phase-contrast MRI has been used to assess regional blood flow parameters such as flow velocity and volume and regurgitant fraction. Recently, fourdimensional (4D) flow MRI that acquires three-dimensional (3D) velocity and encodes volume coverage has been developed and applied to various clinical settings. 4D flow MRI allows retrospective flow measurement at any cross-section and 3D flow visualization through postprocessing. More advanced flow parameters based on fluid dynamics have been proposed and applied to 4D flow MRI to further understand flow mechanisms that might be related to the evolution and progression of cardiovascular diseases. The purpose of this review is to introduce the basics of $4 \mathrm{D}$ flow image acquisition and postprocessing, and its clinical application to selected cardiovascular diseases.
\end{abstract}

Key words Magnetic resonance imaging · Hemodynamics · Heart diseases Vascular diseases $\cdot$ Pulmonary hypertension.

Received: March 7, 2018

Corresponding author Hideki Ota, MD, PhD

Department of Diagnostic Radiology, Tohoku University Hospital,

1-1 Seiryo-machi, Aoba-ku, Sendai,

Miyagi 980-8574, Japan

Tel: 81-22-717-7312

Fax: 81-22-717-7316

E-mail: h-ota@rad.med.tohoku.ac.jp

\section{INTRODUCTION}

Magnetic resonance imaging (MRI) has become an important diagnostic tool for evaluating the cardiovascular system. Various image acquisition protocols are conducted to diagnose cardiac and vascular diseases, measure volumes and functions, and assess the effects of medical, endovascular, and surgical interventions in routine clinical settings. Among them, phase-contrast (PC) MRI has been mainly used for two purposes since the early clinical application of MRI in the 1980s [1,2]. The first purpose is two-dimensional (2D)/three-dimensional (3D) PC magnetic resonance angiography (PC MRA) using its magnitude image. This technique has been applied to various fields from the cerebrovascular system to the lower peripheral arteries [3-6]. The second purpose is 2D PC MRI for flow quantification based on the direct relationship between the phase of MR signals and

(c) This is an Open Access article distributed under the terms of the Creative Commons Attribution Non-Commercial License (http://creativecommons.org/licenses/bync/4.0) which permits unrestricted non-commercial use, distribution, and reproduction in any medium, provided the original work is properly cited. flow velocity. The imaging plane is generally set orthogonal to the vascular axis, providing various flow-related parameters such as blood flow velocity, blood flow volume, and regurgitant fraction through the cross-sections to evaluate the hemodynamic state.

Recently, time-resolved PC MRI with velocity encoding along $\mathrm{x}-, \mathrm{y}-$, and $\mathrm{z}$-directions and 3D volumetric anatomic coverage [also termed four-dimensional (4D) flow MRI] has been developed and applied for the evaluation of cardiovascular hemodynamics in multiple regions of the human body [7]. 4D flow MRI has several advantages over conventional 2D PC MRI, such as measuring velocity parameters on any plane placed retrospectively at postprocessing, 3D flow visualization, and advanced hemodynamic parameters using $3 \mathrm{D}$ velocity information.

The purpose of this review is to introduce the basic protocols, advantages and disadvantages, and postprocessing for image evaluation of 4D flow MRI. A selected clinical application of $4 \mathrm{D}$ flow MRI will also be illustrated and further potential applications of 4D flow MRI as a cardiovascular imaging modali- 
ty will be discussed.

\section{IMAGE ACQUISITION AND POSTPROCESSING}

\section{Blood flow velocity measurement on 2D PC MRI}

On PC MRI, magnetic field gradients are used to encode the spatial movement of the spins. Sequence schemes are characterized by velocity encoded bipolar gradients consisting of two lobes of equal area (duration and amplitude) and opposite gradient polarity similar to the diffusion-weighted sequences $[8,9]$. The bipolar gradients generate phase shifts, resulting in net-zero shifts for stationary spins. However, moving spins gain a nonzero phase because the encoding magnetic field strength changes with the location of the moving spins. If the spins move along the direction of the gradient with a constant velocity, the change in the phase is proportional to the velocity of the moving spins. The velocity information can be obtained as a phase-difference image, which is created by subtraction of two phase images acquired by toggling the bipolar gradient (acquisition of all imaging parameters is the same except for the polarity of the bipolar gradient) or acquired with and without velocity encoding. For accurate velocity quantification, it is important to assess background phase errors caused by the eddy current, concomitant gradient field, and gradient non-linearity [10]. Generally, the 2D PC cross-sectional image is set perpendicular to the flow direction to obtain through-plane velocity [11]. Acquisition of data over multiple cardiac cycles generates a series of flow velocity (phase difference) and anatomical (magnitude) images reflecting the temporal changes in velocity and morphology in the target vessel [7].

One of the most important acquisition parameters in PC MRI is the velocity encoding setting [7]. Velocity encoding is defined as the speed (usually in centimeters per second) along the bipolar gradient that provides the phase difference of $\pi$ $\left(180^{\circ}\right)$. A velocity encoding setting lower than the maximum flow velocity results in a large phase difference exceeding $\pm \pi$ and aliasing artifacts (between $\pi+\alpha$ and $-\pi+\alpha$ ) [11]. In contrast, a velocity encoding setting that is too high causes small phase differences and a low velocity-to-noise ratio. Therefore, it is ideal to set the velocity encoding as low as possible yet not below the maximum velocity [7]. Antialiasing correction (phase unwrapping) may be possible at postprocessing and implemented in some software. The suggested velocity encoding settings are $150-200 \mathrm{~cm} / \mathrm{s}$ for the thoracic aorta, $250-400 \mathrm{~cm} / \mathrm{s}$ for aortic valve stenosis and coarctation of the aorta, $100-150 \mathrm{~cm} / \mathrm{s}$ for intracardiac blood flow, and $50-80 \mathrm{~cm} / \mathrm{s}$ for other great arteries and veins [7].

Tracing vessel borders in magnitude images allows measurement of flow-related parameters during the cardiac cycle, such as mean blood flow through the cardiac cycle, blood flow and velocity in each cardiac phase, ejection time, and acceleration time $[12,13]$.

\section{Data acquisition on $4 \mathrm{D}$ flow MRI}

On 4D flow MRI, the abovementioned 2D PC MRI is expanded to 3D acquisition. Velocity encoding along the $\mathrm{x}^{-}, \mathrm{y}^{-}$, and $\mathrm{z}$-directions with $3 \mathrm{D}$ volumetric coverage provides datasets containing $3 \mathrm{D}$ velocity vector in each voxel during cardiac cycles [14]. In the fields of the chest and abdomen, respiratory gating is implemented if necessary. Although the total scan time available for a clinical MRI examination is limited, sufficient spatial ( $>5-6$ voxels across the vessel diameter) and temporal ( $<40 \mathrm{~ms})$ resolutions have been recommended to achieve accurate flow quantification [11].

Magnitude images acquired using 4D flow MRI are termed 3D PC MRA images. The anatomical information of the vascular lumen plays an important role in vascular volume extraction and flow volume quantification in postprocessing of $4 \mathrm{D}$ flow MRI. Some postprocessing software can use other MRA images, such as balanced steady-state free precession (SSFP) images for vessel segmentation [15], although misalignment between the anatomical and velocity images may occur. The potential merits of balanced SSFP images include bright blood images with high signal-to-noise ratio (SNR) [16].

\section{Use of gadolinium-based contrast media}

Theoretically, 4D flow MRI does not require gadoliniumbased contrast media because the signal intensity is dependent on flow velocity. In large vessels with large flow velocities, such as the aorta and its major branches, good SNR, velocity-tonoise ratio, and contrasts between the vessel and surrounding tissues can be acquired without using gadolinium-based contrast media [7]. However, contrast-enhanced 4D flow MRI can take advantage of enhanced SNR and contrasts, which enables visualization of the vessels even when they have a small size and/ or slow blood flow velocity. Changes in the signal intensity during 4D flow scan depending on the timing after administration of contrast media may affect velocity data.

\section{Four-dimensional flow MRI postprocessing and analysis}

\section{Two-dimensional flow analysis on any cross-section}

One of the great advantages of $4 \mathrm{D}$ flow MRI over traditional 2D PC MRI is that the postprocessing allows analysis of any vascular cross-section in the acquired volume data. Therefore, it is easy to tweak the angular alignment along the vascular course or analyze multiple cross-sections without additional scans (Fig. 1). Studies that compared measured blood flow ve- 
locities have demonstrated good agreement between 2D PC and 4D flow MRIs in various fields, such as the abdominal and intracranial arteries $[17,18]$.

\section{Visualization of 3D blood flow}

On 4D flow MRI each voxel has information on the velocity vector. The process of connecting velocity vectors in adjacent voxels can generate $3 \mathrm{D}$ continuity of velocity vectors, which can be visualized using various methods such as instantaneous streamlines and flow vectors (Fig. 2) [7]. Calculating the paths of virtual massless particles provides path lines or particle traces. Various flow patterns in the target vessels, such as laminar flow, helical flow, and vortical flow, are demonstrated in the postprocessed 3D images (Figs. 1-6).

\section{Flow analysis parameters}

4D flow MRI can provide information on conventional flow analysis parameters on vascular cross-sections obtained using 2D PC MRI. These parameters include blood flow velocity, blood flow volume, antegrade and retrograde flows, regurgitant fraction, mean, minimal, and maximal flow velocities, and time-velocity curve. Furthermore, taking advantage of 3D velocity vector information, $4 \mathrm{D}$ flow MRI can be used to obtain more advanced parameters of hemodynamics such as wall shear stress (the tangential frictional force per unit area exerted by the flowing fluid on the luminal surface), oscillatory shear index (the directional change of wall shear stress during the cardiac cycle), kinetic energy (the mass of blood in the voxel multiplied by the square of the velocity in that voxel divided by

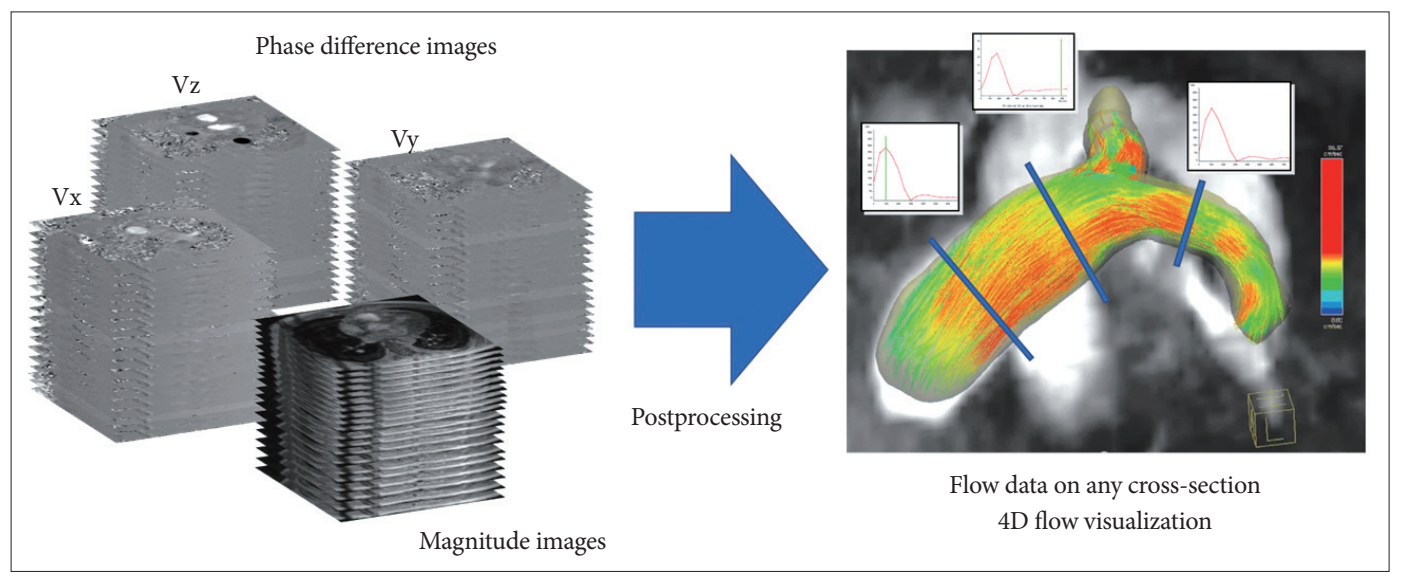

Fig. 1. Schematic illustration of 4D flow MRI. Schematic illustration of 4D flow MRI data acquisition and postprocessing. Four 3D raw datasets for each cardiac phase were collected consisting of magnitude images and three sets of velocity-encoded phase difference images to measure three-directional blood flow velocities $(\mathrm{Vx}, \mathrm{Vy}$, and $\mathrm{Vz}$ ). The datasets were postprocessed using software. The postprocessing included several steps such as background phase correction, phase antialiasing, and segmentation of the target vessels. This method provided 3D flow visualization, retrospective flow quantification at any cross-section, and more advanced flow parameters. 4D flow MRI: fourdimensional flow magnetic resonance imaging, 3D: three-dimensional.
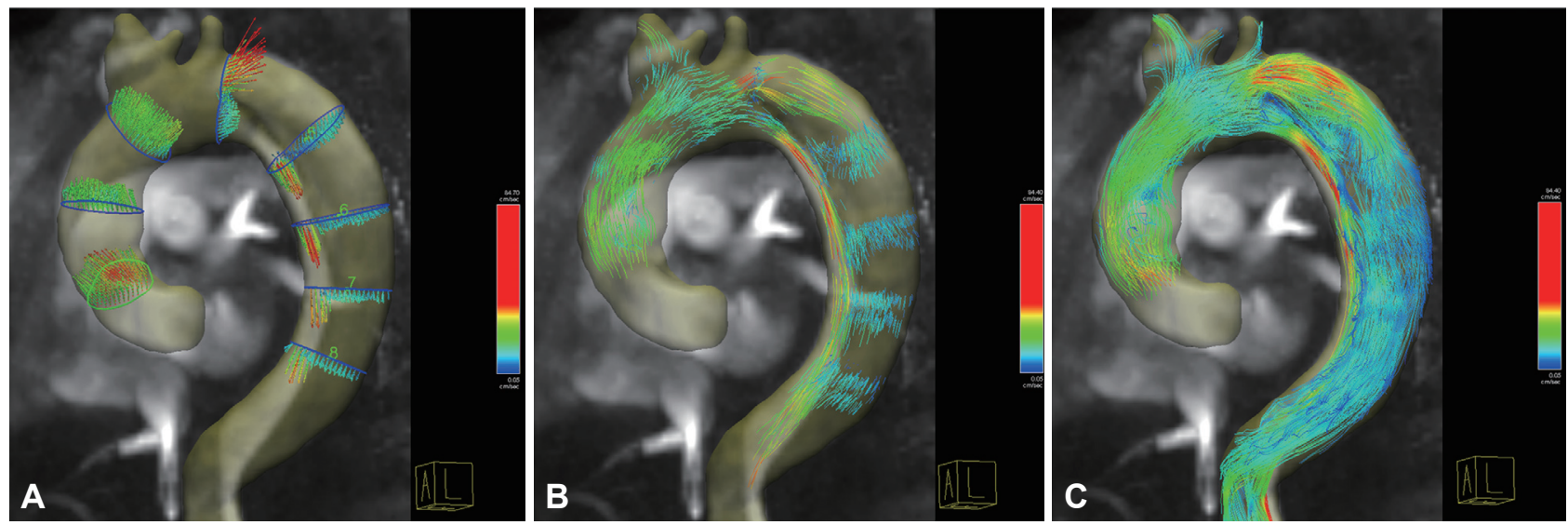

Fig. 2. Stanford type B aortic dissection. The flow velocity into the false lumen was accelerated at the entry site just distal to the ostium of the left subclavian artery. In the descending aorta, the flow velocity appeared to be faster in the true lumen than in the false lumen. (A) Flow velocity vector image in which vectors at multiple cross-sections were demonstrated by their three-dimensional direction, length, and color. The bottom graph demonstrates flow volumes together with cardiac phases at individual cross-sections. (B) Particle trace image in which the particles were seeded at the same locations as in (A). (C) Streamline image representing the trajectories that the particles take through a static vector field. 
Table 1. Selected clinical applications of four-dimensional flow magnetic resonance imaging

\begin{tabular}{ll}
\hline \multicolumn{1}{c}{ Vascular territories } & \multicolumn{1}{c}{ Clinical indications } \\
\hline Cerebral arteries & Extracranial-intracranial bypass [44] \\
& Arteriovenous malformations [45,46] \\
Aorta & Aortic dissection [23,28] \\
& Thoracic aortic aneurysm [29,30] \\
& Endoleak after endovascular aortic repair \\
& {$[31]$} \\
& Retrograde aortic flow as a potential source \\
& of embolic stroke [47] \\
& Bicuspid aortic valve [32,33,48] \\
& Coarctation of the aorta [49] \\
& Pulmonary hypertension [38-40,42] \\
& Intracardiac flow visualization [50] \\
Pulmonary artery & Congenital heart disease [24,34-37] \\
Heart & Cirrhosis [43] and portal hypertension [51] \\
Liver and portal vein & Renal artery stenosis [17] \\
Kidney & Peripheral arterial disease [88] \\
Peripheral arteries &
\end{tabular}

two), vorticity (rotating or swirling motion around an orthogonal axis to the vessel centerline), and helicity (rotational motion around the longitudinal axis of the vessel centerline) [19-22]. Studies have reported the clinical application of these advanced parameters using 4D flow MRI [23-27].

\section{CLINICAL APPLICATION OF 4D FLOW MRI}

Numerous past studies have reported the feasibility and clinical utilities of $4 \mathrm{D}$ flow MRI [23,24,28-51]. Table 1 summarizes the selected clinical applications of $4 \mathrm{D}$ flow MRI in various vascular fields. Past studies have also demonstrated hemodynamics of normal cohorts, various hemodynamic changes in cardiovascular diseases, and comparisons before and after interventions for cardiovascular diseases $[26,41,52,53]$. This article focuses on the clinical application of $4 \mathrm{D}$ flow MRI in the field of the great arteries, including aortic diseases, adult congenital heart diseases (CHDs), and pulmonary hypertension.
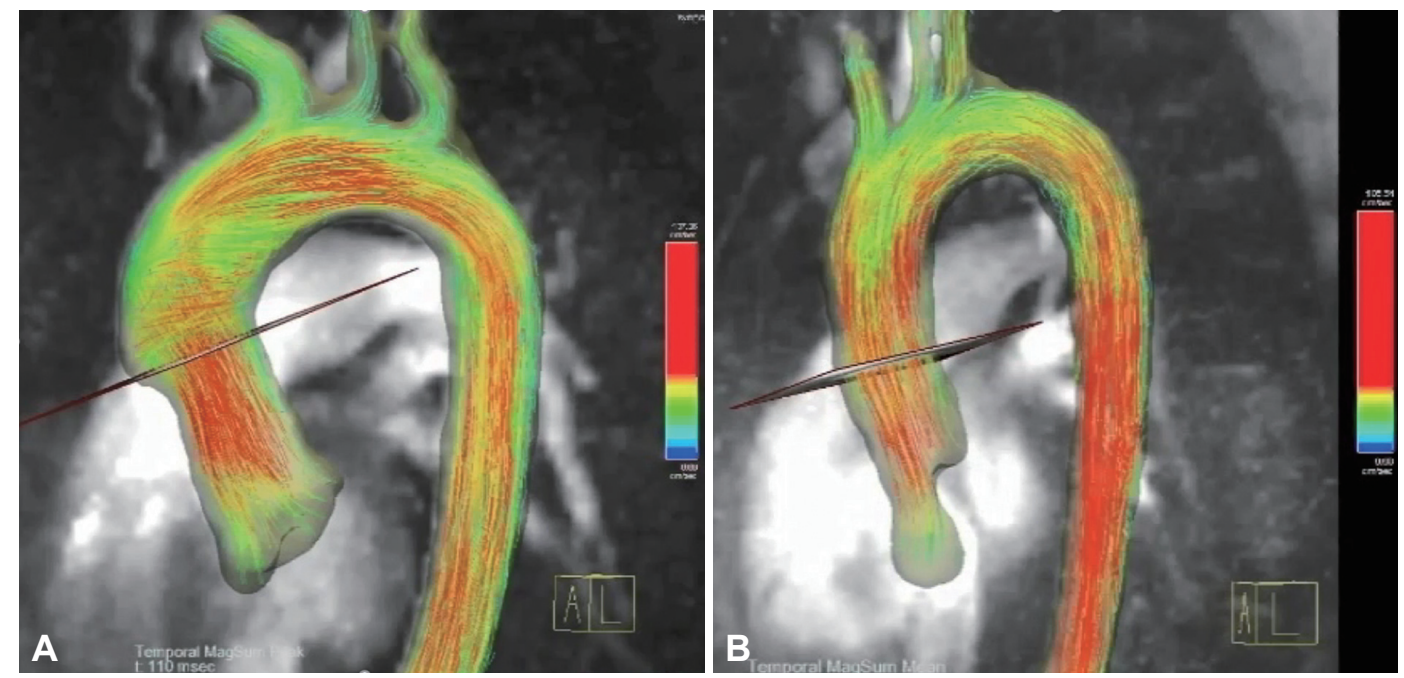

Fig. 3. Four-dimensional flow images of the thoracic aorta. The streamlines in a young subject with a bicuspid valve showed a significant helical flow in the ascending aorta (A) whereas those in a young healthy subject appeared straight (B).
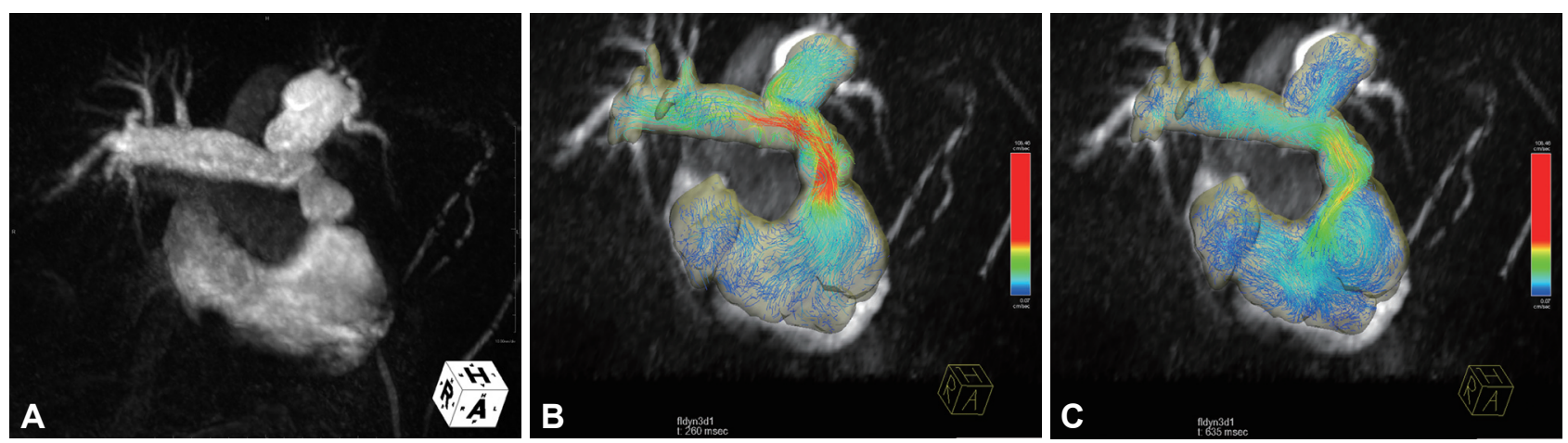

Fig. 4. Repaired tetralogy of Fallot. (A) Maximum intensity projection image of gadolinium contrast-enhanced MR angiography demonstrating residual stenosis at the bifurcation of the pulmonary artery. (B) Particle trace image of 4D flow MRI in the systolic phase demonstrating accelerated flow at the bifurcation. (C) Particle trace image of 4D flow MRI in the diastolic phase demonstrating a regurgitant flow from the main pulmonary artery to the right ventricle and large vortex formation in the right ventricle. MR: magnetic resonance, 4D flow MRI: four-dimensional MR imaging. 


\section{Aortic diseases}

\section{Aortic dissection}

Aortic dissection is a life-threatening emergency condition. A tear in the intimal layer that allows blood flow to propagate within the medial layer generates a false lumen. Any condition that increases intimal shear stress or decreases arterial wall strength is considered to be a risk factor [54]. In contrast to Stanford type A dissection, for which surgical intervention is indicated, medical management is widely accepted as the first-line treatment for uncomplicated type B dissection. However, it is important to perform a risk assessment at an early stage to determine the merits of the intervention [54].

CT is the most commonly used imaging modality for the di-

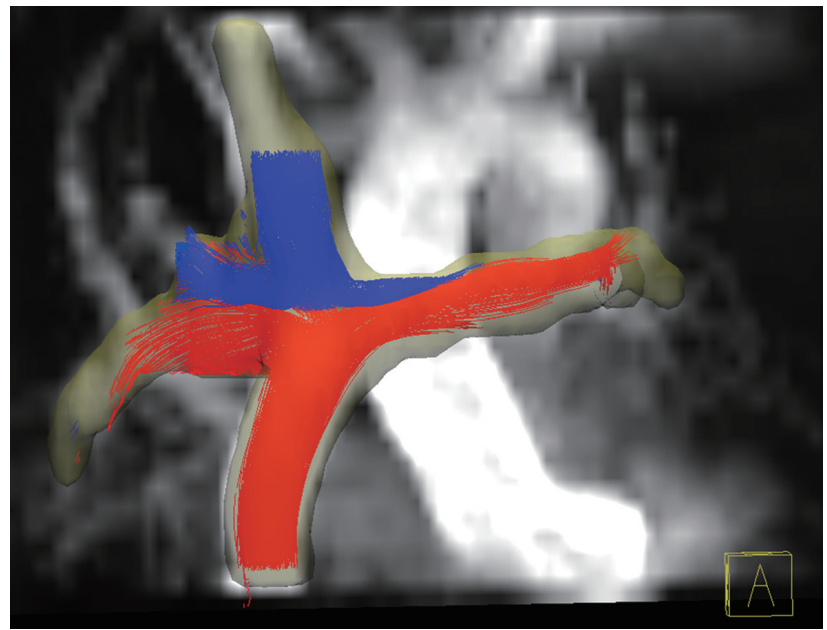

Fig. 5. Particle trace image of four-dimensional flow magnetic resonance imaging in total cavopulmonary connection (anterior view). Blood from both the superior vena cava (blue) and inferior vena cava (red) flowed to the bilateral pulmonary arteries. agnosis and follow-up of aortic dissection because of its speed, widespread availability, and excellent diagnostic accuracy [55]. The diameter of the aorta and the amount of false lumen thrombosis are used to stratify and select patients for treatment [23]. Moreover, recent studies have revealed that hemodynamic parameters, such as flow pattern, flow volume, and velocity, play a role in false lumen expansion $[23,56]$. Although the emerging tool of computed flow dynamics can provide blood flow information, patient-specific inflow boundary conditions may be required to obtain meaningful hemodynamic measurements [57].

MRI has advantages of allowing actual hemodynamic measurements in patients with aortic dissection requiring careful follow-up. Müller-Eschner et al. [58] demonstrated 3D visualization of accelerated blood flow through the entry site towards the false lumen wall in chronic type B aortic dissection using 3D MR velocity mapping (Fig. 2). François et al. [28] used 4D flow MRI to investigate the differences in hemodynamic features between the true lumen and the false lumen in aortic dissection. Their study showed that blood flow was primarily laminar in the true lumen, whereas it appeared complex, abnormal, and nonlaminar in the false lumen. Clough et al. [23] assessed whether $4 \mathrm{D}$ flow MRI can visualize and quantify the flow characteristics of 12 patients with aortic dissection and whether these features are related to the rate of aortic expansion. The stroke volume and velocity in the false lumen were positively associated with more rapid aortic expansion during follow-up. Four-dimensional flow MRI also demonstrated a helical flow in the false lumen in 8 out of 12 patients, which was associated with the rate of aortic expansion [23]. The new features obtained from 4D flow imaging may help provide better predictors for long-term followup, possible complications, and patient mortality and morbidity,
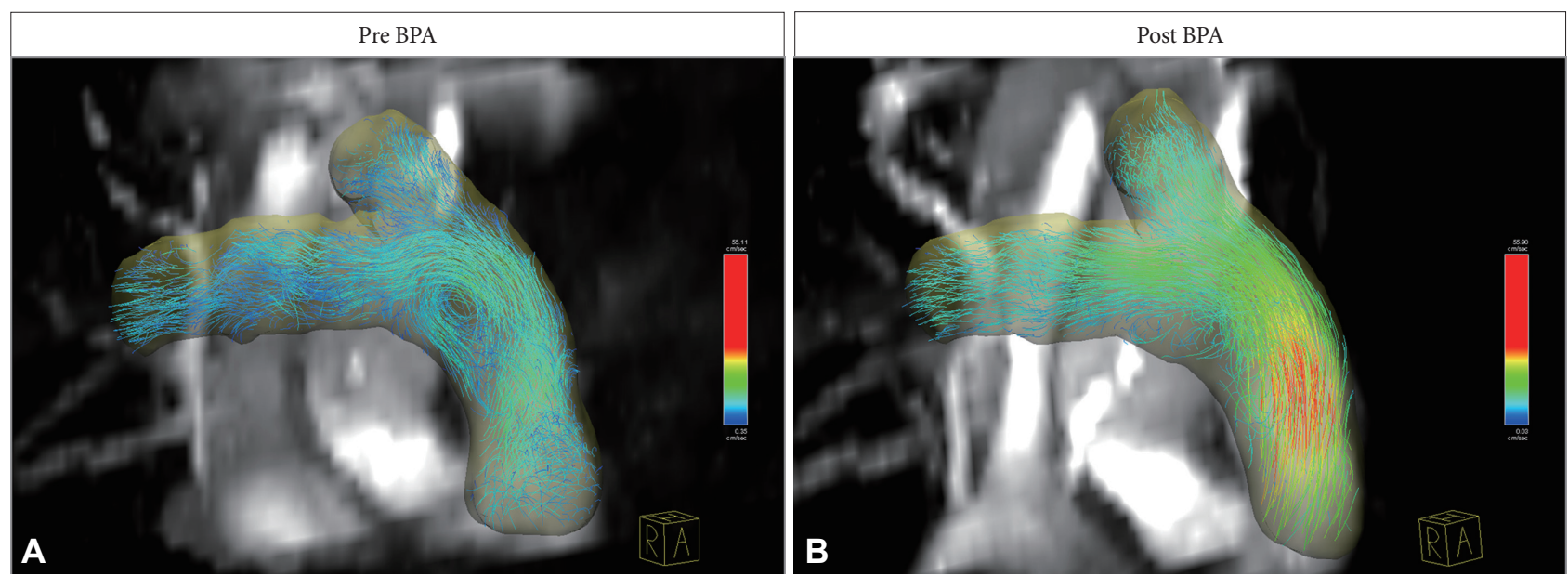

Fig. 6. A patient with chronic thromboembolic pulmonary hypertension underwent four-dimensional flow magnetic resonance imaging before and after BPA. The mean pulmonary artery pressure measured via right heart catheterization improved from $45 \mathrm{~mm} \mathrm{Hg}$ to $24 \mathrm{~mm} \mathrm{Hg}$ after BPA. (A) Particle traces in the end-systolic phase before BPA demonstrating a vortex flow in the distal main pulmonary artery. (B) Particle traces in the end-systolic phase after BPA demonstrating a reduced degree of vortex flow in the distal pulmonary artery. The flow velocity also improved. BPA: balloon pulmonary angioplasty. 
although these associations are yet to be determined.

\section{Aortic aneurysm}

Aortic aneurysm is the second most frequent disease of the aorta after atherosclerosis [55]. Both thoracic aortic aneurysms (TAAs) and abdominal aortic aneurysms (AAAs) require surgical repair for patients with a maximum aortic diameter $\geq 55$ $\mathrm{mm}$ [55]. However, recent data indicated that patients with an aortic size below $50 \mathrm{~mm}$ still have an incremental yearly risk of rupture dissection or death that is greater than 5\% [59]. The current geometric figure datasets may not be fully adequate for preventing acute complications. Disease processes, such as aneurysm formation, are largely dependent on hemodynamic factors in the vascular system.

Past studies have compared the ascending aortic flow characteristics and clinical risk profile of proximal TAAs. Hope et al. [29] compared the flow pattern between healthy volunteers and patients with ascending aortic aneurysms; larger helices, vortex, and retrograde flows were found more often in the patients with aortic aneurysms than in the controls. Kari et al. [30] calculated the flow compression index as a fraction of the area of high-velocity mid-systolic flow over the complete crosssectional ascending aortic aneurysm according to the ascending aortic aneurysm morphology. Eccentric aneurysm was associated with a high level of flow compression (low flow compression index) irrespective of the underlining aortic valve morphology and function. These findings may indicate the potential diagnostic value of $4 \mathrm{D}$ flow MRI in clinical risk evaluation of patients with TAAs [30].

Sakata et al. [31] used 4D flow MRI, computed tomography angiography (CTA), and duplex ultrasonography to identify endoleaks in patients after nitinol-based stent graft deployment for AAAs. Endoleaks were detected by $4 \mathrm{D}$ flow analysis in 18 of 31 (58.1\%) patients, by CTA in 13 patients (41.9\%), and by duplex ultrasonography in six patients (19.4\%), demonstrating that $4 \mathrm{D}$ flow MRI is more sensitive for assessing endoleaks than CTA and ultrasonography. Furthermore, 4D flow MRI could differentiate type IIa endoleaks (to-and-fro biphasic flow pattern forming branch vessels) from type IIb endoleaks (monophasic flow pattern with a connection between the inflow and outflow branches). Their study focused on 4D flow MRI based on its advantages, which enable evaluation of the flow direction as well as the entire view of the endoleaks after endovascular aortic repair.

\section{Adult CHD}

The incidence of CHD varies among studies. Early studies reported an incidence of CHD of approximately 4 to 5 per 1000 live births whereas a later study reported an increasing incidence of up 12 to 14 per 1000 live births [60]. This differ- ence in the incidence (lower in early studies and higher in later studies) reflects the fact that recent development of diagnostic tools such as imaging modalities has allowed the identification of early and mild CHDs, rather than only severe CHD. With great advancements in pediatric cardiology and cardiovascular surgery, most patients with CHD now survive to adulthood and the overall prevalence of adult CHD (ACHD) is estimated to be 3000 per million [61]. Consequently, increasing numbers of patients with ACHD need to be managed with medical, surgical, and endovascular interventions. Because of its noninvasiveness and easy access, transthoracic echocardiography (TTE) is the first-line modality for cardiovascular imaging in ACHD as well as other acquired heart diseases. However, the access window of TTE is limited, especially in the right heart system and pulmonary arteries, despite the importance of assessing right heart function in ACHD [62]. Conventional cardiac MR (CMR) has been used to evaluate complex morphology and function of the heart and cardiovascular hemodynamics. 4D flow MRI may offer a new additional window to evaluate the complex hemodynamics of ACHD. We introduce the current role of CMR and potential 4D flow application of selected ACHDs.

\section{Bicuspid aortic valve}

Bicuspid aortic valve (BAV) is the most common CHD with a reported prevalence of approximately $1 \%$ of all live births [60]. BAV is often identified by a fusion of the two aortic valve cusps with raphe in an area of fusion. Fusion of the left and right coronary cusps is the most common pattern and is reported in over $70 \%$ of BAV cases [63]. Patients with BAV develop valve dysfunctions of aortic stenosis and/or regurgitation and dilated ascending aorta over time. The ascending aorta of patients with BAV enlarges at a higher rate than that of matched controls with tricuspid aortic valves; dilatation of the ascending aorta then becomes a risk factor for aortic dissection and rupture, which are major causes of morbidity and mortality [64]. BAV aortopathy has been attributed to genetic and hemodynamic causes. Although the relative contribution of genetics and hemodynamics remains a subject of debate, both factors are probably contributing factors [65]. The abnormal valve morphology affects the aortic blood flow patterns and velocities, resulting in remodeling of the proximal segment of the aorta from the aortic root to the arch.

$4 \mathrm{D}$ flow imaging is useful for noninvasive evaluation of the altered blood flow pattern and measuring the wall shear stress in the ascending aorta affected by the abnormal morphology and dysfunction of BAV. Past studies have reported an abnormal helical blood flow and significant elevation of the wall shear stress in the ascending aorta of patients with BAV compared with that of individuals with tricuspid valves. Even patients without valve dysfunction and aortic dilatation demon- 
strated these hemodynamic alterations in the ascending aorta (Fig. 3) [32,33].

The pattern of aortic dilatation is dependent on the morphologic valve fusion patterns. Mahadevia et al. [66] reported that the presence and type of BAV fusion were associated with changes in the regional wall shear stress distribution, systolic flow eccentricity, and expression of BAV aortopathy. In the right and left cusp fusion patterns, a flow jet is directed toward the right anterior aortic wall and patients have dilatations in the tubular ascending aorta accompanied by varying degrees of aortic root dilatation. Conversely, in the pattern with fusion of the right and noncoronary cusps, the jet is directed toward the posterior wall of the aorta and dilatation of the aortic root only or involvement of the entire ascending aorta and arch is frequently found.

A recent study reported that valve-mediated hemodynamics are even correlated with the distal ascending aorta [67]. Fourdimensional flow MRI can identify the eccentric jet flow in patients with BAV and may help stratify the risk for development of ascending aortic aneurysms and aortic dissection. Moreover, a current study showed that an elevated aortic wall shear stress corresponded to more severe extracellular matrix dysregulation and elastic fiber degeneration compared with normal wall shear stress of adjacent regions in the same aorta [68]. This indicates that wall shear stress, as assessed using $4 \mathrm{D}$ flow MRI, may serve as a noninvasive biomarker of regional aortic disease in patients with BAV aortopathy.

The majority of patients with BAV will require aortic valve replacement using bioprosthetics or mechanical valves in their lifetime [69]. Recently, the hemodynamic change after aortic valve replacement was evaluated using $4 \mathrm{D}$ flow CMR. Bissell et al. [70] reported that abnormal flow hemodynamics tended to normalize after mechanical aortic valve replacement, in contrast to a remnant abnormal flow pattern after bioprosthetic aortic valve replacement. This indicated that the aortic growth rate after aortic valve replacement might depend on the type of the replaced valve.

Despite the limitation of time and spatial resolution, the parameters evaluated in 4D flow MRI may be novel biomarkers of BAV and might help to stratify the risk of cardiovascular events and predict the prognosis of patients. They may also improve surgical strategies and help develop clinical guidelines that allow individuals to achieve good clinical outcomes [71].

\section{Repaired tetralogy of Fallot}

Repaired tetralogy of Fallot (TOF) is the most common cyanotic CHD and involves four heart defects: a large ventricular septal defect, pulmonary stenosis, right ventricular (RV) hypertrophy, and an overriding aorta. In adults with surgically repaired TOF during childhood, important issues may arise such as en- largement and dysfunction of the right ventricle, pulmonary regurgitation, and stenosis in the right and left pulmonary arteries [62]. A significant amount of pulmonary regurgitation is common after surgical removal of stenosis in the right ventricular outflow tract and pulmonary valve. Although the "free" pulmonary regurgitation can be asymptomatic and tolerated for a long time after the surgical repair, the volume overload results in dilatation of the right ventricle. When the ventricle becomes decompensated, excessive right ventricular dilatation with deteriorated function, arrhythmia, and premature death can be induced. CMR can be used to stratify the risk and surgical intervention of repaired TOF with right heart failure $[52,72,73]$. In clinical practice, the proposed indications of pulmonary valve replacement in asymptomatic patients based on CMR findings include RV end-diastolic volume index $>150 \mathrm{~mL} / \mathrm{m}^{2}$, RV end-systolic volume index $>80 \mathrm{~mL} / \mathrm{m}^{2}$, RV ejection fraction (EF) $<47 \%$, and left ventricular (LV) $\mathrm{EF}<55 \%$ [73].

The application of $4 \mathrm{D}$ flow MRI for repaired TOF includes quantification of the flow volume (regurgitant flow volumes and fraction and flow distribution in the left and right pulmonary arteries and collaterals) and peak velocity and visualization of the flow directionality [11]. 3D visualization of hemodynamics in the entire heart and proximal great arteries provides a comprehensive overview of the flow pattern changes in patients with repaired TOF (Fig. 4) [34]. A study by Geiger et al. [34] comparing comprehensive hemodynamics between patients with repaired TOF and healthy subjects showed approximately 2.6 times higher blood flow in the right pulmonary artery than in the left pulmonary artery in patients with repaired TOF; in contrast, the flow ratio of the right pulmonary artery to the left pulmonary artery was 1.1 in the controls. The systolic peak velocity in the pulmonary trunk was approximately two times higher in the patients than in the controls. Although differential blood flow and regurgitation in the branched pulmonary arteries were discussed earlier for 2D PC MRI [35,74], $4 \mathrm{D}$ flow MRI covers the entire volume thus improving understanding of flow alterations in patients with repaired TOF. Hirtler et al. [24] assessed intracardiac flow and vorticity in patients with repaired TOF, and confirmed the advantage of $4 \mathrm{D}$ flow MRI over conventional 2D PC CMR. The right heart intra-arterial, intraventricular, and outflow tract flow patterns differed significantly between healthy volunteers and affected patients. The peak vorticity in both the right atrium and ventricle was significantly higher in the patients. Moreover, the degree of regurgitant flow in the pulmonary trunk was associated with higher vorticities in the right atrium and ventricle. Jeong et al. [36] assessed differences in ventricular kinetic energy between patients with repaired TOF and healthy volunteers. The peak systolic kinetic energy in both the right and left ventricles appeared to be higher in the patients with repaired TOF than in 
the healthy subjects, although the difference was not significantly different. The ratio of the main pulmonary artery flow to the right ventricular kinetic energy and the ratio of the aortic flow to the left ventricular kinetic energy were both lower in the patients with repaired TOF than in the controls. These results indicate that a greater ventricular kinetic energy is necessary to generate blood flow in the pulmonary and systemic circulations in patients with repaired TOF than in healthy subjects.

4D flow MRI in repaired TOF visualizes abnormal flow dynamics and helps us understand and explore new parameters, such as flow energy loss, that may potentially be used as imaging biomarkers [37,75]. Comprehensive coverage of flow velocity data in $4 \mathrm{D}$ flow MRI may reveal interactions of functional and hemodynamic findings associated with right heart failure in repaired TOF. Future studies are warranted to assess whether such parameters derived from $4 \mathrm{D}$ flow MRI allow determination of surgical interventions or risk stratification.

\section{Fontan circulation for functionally single ventricles}

Various congenital complex cardiac defects are associated with only single functional ventricles, which have to maintain both the systemic and pulmonary blood circulations. In "Fontan circulation," the systemic venous return is connected to the pulmonary arteries without the interposition of the ventricle to improve arterial desaturation and chronic volume overload [76]. One of the Fontan circulations is total cavopulmonary connection where the inferior vena cava is connected to the pulmonary artery, bypassing the right atrium through the extracardiac conduit, and the superior vena cava is directly connected to the pulmonary artery. Patients with single ventricle diseases are now mainly treated with total cavopulmonary connection and the survival rate of these patients has improved over the past decades. However, most patients develop a late complication such as pulmonary arteriovenous malformation, systemic ventricular dysfunction, or thromboembolism, due to the abnormal hemodynamics.

$4 \mathrm{D}$ flow MRI can evaluate the eccentric blood flow, which is difficult to assess using echocardiography (Fig. 5). A recent study revealed asymmetric caval blood flow distribution to the right and left pulmonary arteries in patients with Fontan circulation [77]. 4D flow MRI is also used to quantify the kinetic energy in the intraventricular blood flow and might be a useful noninvasive method of monitoring patients with Fontan circulation and detecting signs of ventricular and circulatory dysfunctions [78].

\section{Pulmonary hypertension}

Pulmonary hypertension is defined as a mean pulmonary artery pressure (mPAP) at rest of $>25 \mathrm{~mm} \mathrm{Hg}$ measured via invasive right heart catheterization. The clinical symptoms of pul- monary hypertension include dyspnea upon exertion, fatigue, chest pain, and syncope that limits physical activities. Although these clinical symptoms are non-specific for pulmonary hypertension, their severity is associated with mortality and morbidity. TTE is widely used for the screening of pulmonary hypertension as a noninvasive imaging modality; however, its specificity for identifying pulmonary hypertension is limited [79]. Therefore, $4 \mathrm{D}$ flow MRI may have a promising potential to identify and evaluate the severity of pulmonary hypertension and subsequently monitor the therapeutic effects. Pulmonary hypertension is classified into five major groups, which share similar pathological and hemodynamic characteristics and therapeutic approaches [80].

An elevation in pulmonary arterial pressure and pulmonary vascular resistance alter the blood flow patterns in the major pulmonary arteries. Past studies have used several blood flow parameters in the main pulmonary artery to characterize pulmonary hypertension [38-40,81]. Reiter et al. [39] demonstrated the appearance of vortical flow in the main pulmonary artery on time-resolved 3D PC MRI in patients with pulmonary hypertension. The manifestations of pulmonary hypertension coincided with the appearance of vortical blood flow in the main pulmonary artery with high sensitivity and specificity. They also demonstrated that the duration of vortical blood flow (percentage of the cardiac phases with the vortex present, $\left.t_{\text {vortex }}\right)$ in the main pulmonary artery as observed on 3D PC MRI allowed estimation of elevated mPAPs and diagnosis of pulmonary hypertension [40]. When the cutoff value of $t_{\text {vortex }}$ was set to $14.3 \%$, the sensitivity and specificity to diagnose pulmonary hypertension were $97 \%$ and $96 \%$, respectively [40]. The main pulmonary artery of patients with pulmonary hypertension shows secondary morphological changes including dilatation that may induce vortical formation of the blood flow. In a general population-based study using 4D flow MRI, abnormal vortices occurred in the main pulmonary artery in 3\% of subjects with no history of pulmonary hypertension [41]. The geometry of the main pulmonary artery dividing into the bilateral pulmonary arteries may influence the formation of an abnormal vortical flow even in healthy subjects. Barker et al. [38] used 4D flow MRI to measure the diameter, peak systolic velocity, peak flow, stroke volume, and wall shear stress at the main, right, and left pulmonary arteries in 17 subjects with pulmonary arterial hypertension and 19 healthy subjects. The peak systolic velocity, peak flow, stroke volume, and wall shear stress at all locations were significantly lower in the patients with pulmonary arterial hypertension than in the healthy subjects. Similarly, other investigators reported vortex flow formation, early onset of retrograde flow, and low wall shear stress in the pulmonary artery observed using 4D flow MRI as characteristics of pulmonary hypertension [82]. 
Among the categories of pulmonary hypertension [80], chronic thromboembolic pulmonary hypertension (CTEPH) is unique in its potential for curative interventions. CTEPH develops in $0.1-8.8 \%$ of patients with acute pulmonary embolisms [83]. However, in the clinical setting we often encounter patients without a clear history of pulmonary embolism therefore the exact incidence of CTEPH has been difficult to estimate. Regarding the mechanism, organizing clot and secondary vascular remodeling in the pulmonary arteries induce elevation of pulmonary vascular resistance resulting in pulmonary hypertension [84]. According to guidelines $[85,86]$, pulmonary thromboendarterectomy and newly developed balloon angioplasty combined with medications can be indicated to treat CTEPH. We evaluated the degree of vortex flow in the main pulmonary artery using the ratio of retrograde flow area to the arterial cross-sectional area where the maximum vortex flow was observed in the end-systolic phase. The ratio of the retrograde flow in the main pulmonary artery was moderately correlated with the mPAP in patients with CTEPH $(r=0.68, \mathrm{p}<0.01)$ [42]. Furthermore, the degree of vortex flow decreased after several balloon pulmonary angioplasty (BPA) sessions together with improvement of the mPAP (Fig. 6) [53]. This indicates that $4 \mathrm{D}$ flow MRI can potentially monitor the effects of treatment in patients with CTEPH who undergo BPA.

\section{CURRENT LIMITATIONS AND FUTURE DIRECTIONS OF 4D FLOW MRI}

\section{Long scan time}

As shown in Fig. 1, a long scan time is generally required to acquire a large amount of volume datasets. Therefore, the balance between acquisition volume, spatial resolution, and time resolution should be taken into account to complete clinical examinations within reasonable time slots. However, accelerated image acquisition has been improving due to the development of hardware and data acquisition algorithms; new technologies that can be implemented for 4D flow MRI include highperformance gradient system enabling shorter echo time and repetition time, phased-array coils, multi-channel receiving system, parallel imaging, compressed sensing, and radial sampling [43]. Respiratory motions should also be considered when imaging the thoracic and abdominal vascular systems and use of abdominal compression belts is a common method to minimize respiratory motions and thus increase the acceptance rate of respiratory gating during acquisition. Real-time respiratory selfgating is also possible for 4D flow imaging [87].

\section{Postprocessing}

Currently, several third-party venders provide commercial software separate from the MR system manufacturers. Report- ed studies have used in-house programs and commercial products for postprocessing. Accessibility is one of the key factors for widespread use of 4D flow MRI in the clinical setting. A cloud-based postprocessing system has been launched, which may accelerate the clinical application of $4 \mathrm{D}$ flow MRI. Although numerous studies have been conducted to demonstrate its utility in congenital and acquired cardiovascular diseases, accumulation of more evidence is required to establish the clinical role of $4 \mathrm{D}$ flow MRI as an emerging technique for patient management and risk stratification.

\section{SUMMARY}

$4 \mathrm{D}$ flow MRI can be used to analyze 3D hemodynamics based on the velocity vector, with potential clinical application in various fields. The retrospective setting of analysis planes for blood flow evaluation is a large advantage of $4 \mathrm{D}$ flow MRI in the clinical setting. The 3D flow visualization offers comprehensive understanding of flow fields. Advanced flow parameters have been proposed for comprehensive analysis of flow dynamics. Although these parameters are currently mainly applied in research fields, they may play a role in revealing the mechanisms underlying the evolution and progression of cardiovascular diseases.

\section{Conflicts of Interest}

The authors declare that they have no conflict of interest.

\section{Acknowledgments}

This work was supported by JSPS KAKENHI Grant Number 16K10265.

\section{REFERENCES}

1. Moran PR. A flow velocity zeugmatographic interlace for NMR imaging in humans. Magn Reson Imaging 1982;1:197-203.

2. Bryant DJ, Payne JA, Firmin DN, Longmore DB. Measurement of flow with NMR imaging using a gradient pulse and phase difference technique. J Comput Assist Tomogr 1984;8:588-593.

3. Huston J 3rd, Rufenacht DA, Ehman RL, Wiebers DO. Intracranial aneurysms and vascular malformations: comparison of time-of-flight and phase-contrast MR angiography. Radiology 1991;181:721-730.

4. Pernicone JR, Siebert JE, Potchen EJ, Pera A, Dumoulin CL, Souza SP. Three-dimensional phase-contrast MR angiography in the head and neck: preliminary report. AJR Am J Roentgenol 1990;155:167-176.

5. Steffens JC, Link J, Müller-Hülsbeck S, Freund M, Brinkmann G, Heller M. Cardiac-gated two-dimensional phase-contrast MR angiography of lower extremity occlusive disease. AJR Am J Roentgenol 1997;169:749754.

6. Prince MR, Schoenberg SO, Ward JS, Londy FJ, Wakefield TW, Stanley JC. Hemodynamically significant atherosclerotic renal artery stenosis: MR angiographic features. Radiology 1997;205:128-136.

7. Stankovic Z, Allen BD, Garcia J, Jarvis KB, Markl M. 4D flow imaging with MRI. Cardiovasc Diagn Ther 2014;4:173-192.

8. Bernstein MA, King KF, Zhou XJ. Angiographic pulse sequence. In: Bernstein MA, King KF, Zhou XJ, ed. Handb MRI pulse sequences. Burlington: Academic Press, 2004:648-701.

9. Wheaton AJ, Miyazaki M. Non-contrast enhanced MR angiography: 
physical principles. J Magn Reson Imaging 2012;36:286-304.

10. Busch J, Giese D, Kozerke S. Image-based background phase error correction in 4D flow MRI revisited. J Magn Reson Imaging JMRI 2017; 46:1516-1525.

11. Dyverfeldt P, Bissell M, Barker AJ, Bolger AF, Carlhäll CJ, Ebbers T, et al. 4D flow cardiovascular magnetic resonance consensus statement. J Cardiovasc Magn Reson 2015;17:72.

12. Schulz-Menger J, Bluemke DA, Bremerich J, Flamm SD, Fogel MA, Friedrich MG, et al. Standardized image interpretation and post processing in cardiovascular magnetic resonance: Society for Cardiovascular Magnetic Resonance (SCMR) board of trustees task force on standardized post processing. J Cardiovasc Magn Reson 2013;15:35.

13. Roeleveld RJ, Marcus JT, Boonstra A, Postmus PE, Marques KM, Bronzwaer JG, et al. A comparison of noninvasive MRI-based methods of estimating pulmonary artery pressure in pulmonary hypertension. J Magn Reson Imaging 2005;22:67-72.

14. Burris NS, Hope MD. 4D Flow MRI applications for aortic disease. Magn Reson Imaging Clin N Am 2015;23:15-23.

15. Miyazaki S, Itatani K, Furusawa T, Nishino T, Sugiyama M, Takehara Y, et al. Validation of numerical simulation methods in aortic arch using 4D flow MRI. Heart Vessels 2017;32:1032-1044.

16. Miyazaki M, Lee VS. Nonenhanced MR angiography. Radiology 2008; 248:20-43.

17. Wentland AL, Grist TM, Wieben O. Repeatability and internal consistency of abdominal 2D and 4D phase contrast MR flow measurements. Acad Radiol 2013;20:699-704.

18. Meckel S, Leitner L, Bonati LH, Santini F, Schubert T, Stalder AF, et al. Intracranial artery velocity measurement using $4 \mathrm{D}$ PC MRI at $3 \mathrm{~T}$ : comparison with transcranial ultrasound techniques and 2D PC MRI. Neuroradiology 2013;55:389-398.

19. Grus T, Lambert L, Matěcha J, Grusová G, Špaček M, Mlček M. The ratio of diameters between the target artery and the bypass modifies hemodynamic parameters related to intimal hyperplasia in the distal end-to-side anastomosis. Physiol Res 2016;65:901-908.

20. Carlsson M, Heiberg E, Toger J, Arheden H. Quantification of left and right ventricular kinetic energy using four-dimensional intracardiac magnetic resonance imaging flow measurements. Am J Physiol Heart Circ Physiol 2012;302:H893-H900.

21. Fenster BE, Browning J, Schroeder JD, Schafer M, Podgorski CA, Smyser $\mathrm{J}$, et al. Vorticity is a marker of right ventricular diastolic dysfunction. Am J Physiol-Heart Circ Physiol 2015;309:H1087-H1093.

22. Saba L. Imaging of the cardiovascular system, thorax, and abdomen. Boca Raton (FL): CRC Press, 2017.

23. Clough RE, Waltham M, Giese D, Taylor PR, Schaeffter T. A new imaging method for assessment of aortic dissection using four-dimensional phase contrast magnetic resonance imaging. J Vasc Surg 2012;55:914-923.

24. Hirtler D, Garcia J, Barker AJ, Geiger J. Assessment of intracardiac flow and vorticity in the right heart of patients after repair of tetralogy of Fallot by flow-sensitive 4D MRI. Eur Radiol 2016;26:3598-3607.

25. Markl M, Wegent F, Zech T, Bauer S, Strecker C, Schumacher M, et al. In vivo wall shear stress distribution in the carotid artery: effect of bifurcation geometry, internal carotid artery stenosis, and recanalization therapy. Circ Cardiovasc Imaging 2010;3:647-655.

26. van der Geest RJ, Garg P. Advanced analysis techniques for intra-cardiac flow evaluation from 4D flow MRI. Curr Radiol Rep 2016;4:38.

27. Crandon S, Elbaz MSM, Westenberg JJM, van der Geest RJ, Plein S, Garg P. Clinical applications of intra-cardiac four-dimensional flow cardiovascular magnetic resonance: a systematic review. Int J Cardiol 2017;249:486493.

28. François CJ, Markl M, Schiebler ML, Niespodzany E, Landgraf BR, Schlensak C, et al. Four-dimensional, flow-sensitive magnetic resonance imaging of blood flow patterns in thoracic aortic dissections. J Thorac Cardiovasc Surg 2013;145:1359-1366.

29. Hope TA, Markl M, Wigström L, Alley MT, Miller DC, Herfkens RJ. Comparison of flow patterns in ascending aortic aneurysms and volun- teers using four-dimensional magnetic resonance velocity mapping. J Magn Reson Imaging 2007;26:1471-1479.

30. Kari FA, Kocher N, Beyersdorf F, Tscheuschler A, Meffert P, Rylski B, et al. Four-dimensional magnetic resonance imaging-derived ascending aortic flow eccentricity and flow compression are linked to aneurysm morphology. Interact Cardiovasc Thorac Surg 2015;20:582-588.

31. Sakata M, Takehara Y, Katahashi K, Sano M, Inuzuka K, Yamamoto N, et al. Hemodynamic analysis of endoleaks after endovascular abdominal aortic aneurysm repair by using 4-dimensional flow-sensitive magnetic resonance imaging. Circ J 2016;80:1715-1725.

32. Barker AJ, Markl M, Bürk J, Lorenz R, Bock J, Bauer S, et al. Bicuspid aortic valve is associated with altered wall shear stress in the ascending aortaclinical perspective. Circ Cardiovasc Imaging 2012;5:457-466.

33. Meierhofer C, Schneider EP, Lyko C, Hutter A, Martinoff S, Markl M, et al. Wall shear stress and flow patterns in the ascending aorta in patients with bicuspid aortic valves differ significantly from tricuspid aortic valves: a prospective study. Eur Heart J Cardiovasc Imaging 2013;14:797-804.

34. Geiger J, Markl M, Jung B, Grohmann J, Stiller B, Langer M, et al. 4DMR flow analysis in patients after repair for tetralogy of Fallot. Eur Radiol 2011;21:1651-1657.

35. Kang IS, Redington AN, Benson LN, Macgowan C, Valsangiacomo ER, Roman $\mathrm{K}$, et al. Differential regurgitation in branch pulmonary arteries after repair of tetralogy of Fallot: a phase-contrast cine magnetic resonance study. Circulation 2003;107:2938-2943

36. Jeong D, Anagnostopoulos PV, Roldan-Alzate A, Srinivasan S, Schiebler $\mathrm{ML}$, Wieben $\mathrm{O}$, et al. Ventricular kinetic energy may provide a novel noninvasive way to assess ventricular performance in patients with repaired tetralogy of Fallot. J Thorac Cardiovasc Surg 2015;149:1339-1347.

37. Shibata M, Itatani K, Hayashi T, Honda T, Kitagawa A, Miyaji K, et al. Flow energy loss as a predictive parameter for right ventricular deterioration caused by pulmonary regurgitation after tetralogy of Fallot repair. Pediatr Cardiol 2018 Feb 16 [Epub]. https://doi.org/10.1007/s00246-0181813-z.

38. Barker AJ, Roldán-Alzate A, Entezari P, Shah SJ, Chesler NC, Wieben O, et al. Four-dimensional flow assessment of pulmonary artery flow and wall shear stress in adult pulmonary arterial hypertension: results from two institutions. Magn Reson Med 2015;73:1904-1913.

39. Reiter G, Reiter U, Kovacs G, Kainz B, Schmidt K, Maier R, et al. Magnetic resonance-derived 3-dimensional blood flow patterns in the main pulmonary artery as a marker of pulmonary hypertension and a measure of elevated mean pulmonary arterial pressure. Circ Cardiovasc Imaging 2008;1:23-30.

40. Reiter G, Reiter U, Kovacs G, Olschewski H, Fuchsjäger M. Blood flow vortices along the main pulmonary artery measured with MR imaging for diagnosis of pulmonary hypertension. Radiology 2015;275:71-79.

41. Wehrum T, Hagenlocher P, Lodemann T, Vach W, Dragonu I, Hennemuth A, et al. Age dependence of pulmonary artery blood flow measured by $4 \mathrm{D}$ flow cardiovascular magnetic resonance: results of a population-based study. J Cardiovasc Magn Reson 2016;18:31.

42. Ota H, Sugimura K, Sato H, Nochioka K, Tatebe S, Yamamoto S, et al. $4 \mathrm{D}$ flow MRI to monitor mean pulmonary arterial pressure in patients with chronic thromboembolic pulmonary hypertension treated by percutaneous transluminal pulmonary angioplasty. Proc Intl Soc Mag Reson Med 23rd Ann Meet Exhib: 2015 May 30-June 5; Toronto, Ontario, Canada.

43. Stankovic Z. Four-dimensional flow magnetic resonance imaging in cirrhosis. World J Gastroenterol 2016;22:89-102.

44. Sekine T, Takagi R, Amano Y, Murai Y, Orita E, Matsumura Y, et al. 4D flow MRI assessment of extracranial-intracranial bypass: qualitative and quantitative evaluation of the hemodynamics. Neuroradiology 2016;58: 237-244.

45. Wu C, Ansari SA, Honarmand AR, Vakil P, Hurley MC, Bendok BR, et al. Evaluation of $4 \mathrm{D}$ vascular flow and tissue perfusion in cerebral arteriovenous malformations: influence of Spetzler-Martin grade, clinical presentation, and AVM risk factors. Am J Neuroradiol 2015;36:1142-1149.

46. Schnell S, Ansari SA, Vakil P, Wasielewski M, Carr ML, Hurley MC, et al. 
Three-dimensional hemodynamics in intracranial aneurysms: influence of size and morphology. J Magn Reson Imaging 2014;39:120-131.

47. Wehrum T, Dragonu I, Strecker C, Schuchardt F, Hennemuth A, Drexl J, et al. Aortic atheroma as a source of stroke - assessment of embolization risk using 3D CMR in stroke patients and controls. J Cardiovasc Magn Reson 2017;19:67.

48. Hope MD, Hope TA, Meadows AK, Ordovas KG, Urbania TH, Alley MT, et al. Bicuspid aortic valve: four-dimensional MR evaluation of ascending aortic systolic flow patterns. Radiology 2010;255:53-61.

49. Rengier F, Delles M, Eichhorn J, Azad YJ, von Tengg-Kobligk H, Ley-Zaporozhan J, et al. Noninvasive pressure difference mapping derived from $4 \mathrm{D}$ flow MRI in patients with unrepaired and repaired aortic coarctation. Cardiovasc Diagn Ther 2014;4:97-103.

50. Rodriguez Muñoz D, Markl M, Moya Mur JL, Barker A, FernándezGolfín C, Lancellotti P, et al. Intracardiac flow visualization: current status and future directions. Eur Heart J Cardiovasc Imaging 2013;14:10291038.

51. Roldán-Alzate A, Frydrychowicz A, Niespodzany E, Landgraf BR, Johnson $\mathrm{KM}$, Wieben $\mathrm{O}$, et al. In vivo validation of $4 \mathrm{D}$ flow MRI for assessing the hemodynamics of portal hypertension. J Magn Reson Imaging JMRI 2013;37:1100-1108.

52. Heng EL, Gatzoulis MA, Uebing A, Sethia B, Uemura H, Smith GC, et al. Immediate and midterm cardiac remodeling after surgical pulmonary valve replacement in adults with repaired tetralogy of Fallot: a prospective cardiovascular magnetic resonance and clinical study. Circulation 2017;136:1703-1713.

53. Ota H, Sugimura K, Miura M, Shimokawa H. Four-dimensional flow magnetic resonance imaging visualizes drastic change in vortex flow in the main pulmonary artery after percutaneous transluminal pulmonary angioplasty in a patient with chronic thromboembolic pulmonary hypertension. Eur Heart J 2015;36:1630.

54. Writing Committee, Riambau V, Böckler D, Brunkwall J, Cao P, Chiesa R, Coppi G, et al.: Editor's Choice-Management of descending thoracic aorta diseases: clinical practice guidelines of the European Society for Vascular Surgery (ESVS). Eur J Vasc Endovasc Surg 2017;5:4-52.

55. Erbel R, Aboyans V, Boileau C, Bossone E, Bartolomeo RD, Eggebrecht H, et al. 2014 ESC Guidelines on the diagnosis and treatment of aortic diseases: document covering acute and chronic aortic diseases of the thoracic and abdominal aorta of the adult. The Task Force for the Diagnosis and Treatment of Aortic Diseases of the European Society of Cardiology (ESC). Eur Heart J 2014;35:2873-2926.

56. Cheng Z, Tan FPP, Riga CV, Bicknell CD, Hamady MS, Gibbs RG, et al. Analysis of flow patterns in a patient-specific aortic dissection model. J Biomech Eng 2010;132:051007.

57. Youssefi P, Sharma R, Figueroa CA, Jahangiri M. Functional assessment of thoracic aortic aneurysms-the future of risk prediction? Br Med Bull 2017;121:61-71.

58. Müller-Eschner M, Rengier F, Partovi S, Unterhinninghofen R, Böckler D, Ley S, et al. Tridirectional phase-contrast magnetic resonance velocity mapping depicts severe hemodynamic alterations in a patient with aortic dissection type Stanford B. J Vasc Surg 2011;54:559-562.

59. Elefteriades JA, Farkas EA. Thoracic aortic aneurysm clinically pertinent controversies and uncertainties. J Am Coll Cardiol 2010;55:841-857.

60. Hoffman JI, Kaplan S. The incidence of congenital heart disease. J Am Coll Cardiol 2002;39:1890-1900.

61. van der Bom T, Bouma BJ, Meijboom FJ, Zwinderman AH, Mulder BJ. The prevalence of adult congenital heart disease, results from a systematic review and evidence based calculation. Am Heart J 2012;164:568-575.

62. Kilner PJ. Imaging congenital heart disease in adults. Br J Radiol 2011;84 Spec No 3:S258-S268.

63. Kiefer TL, Wang A, Hughes GC, Bashore TM. Management of patients with bicuspid aortic valve disease. Curr Treat Options Cardiovasc Med 2011;13:489-505.

64. Losenno KL, Goodman RL, Chu MWA. Bicuspid aortic valve disease and ascending aortic aneurysms: gaps in knowledge. Cardiol Res Pract
2012;2012:145202.

65. Verma S, Siu SC. Aortic dilatation in patients with bicuspid aortic valve. N Engl J Med 2014;370:1920-1929.

66. Mahadevia R, Barker AJ, Schnell S, Entezari P, Kansal P, Fedak PW, et al. Bicuspid aortic cuspusion morphology alters aortic three-dimensional outflow patterns, wall shear stress, and expression of aortopathy. Circulation 2014;129:673-682.

67. Raghav V, Barker AJ, Mangiameli D, Mirabella L, Markl M, Yoganathan AP. Valve mediated hemodynamics and their association with distal ascending aortic diameter in bicuspid aortic valve subjects. J Magn Reson Imaging 2018;47:246-254.

68. Guzzardi DG, Barker AJ, van Ooij P, Malaisrie SC, Puthumana JJ, Belke $\mathrm{DD}$, et al. Valve-related hemodynamics mediate human bicuspid aortopathy: insights from wall shear stress mapping. J Am Coll Cardiol 2015;66: 892-900.

69. Michelena HI, Desjardins VA, Avierinos JF, Russo A, Nkomo VT, Sundt TM, et al. Natural history of asymptomatic patients with normally functioning or minimally dysfunctional bicuspid aortic valve in the community. Circulation 2008;117:2776-2784.

70. Bissell MM, Loudon M, Hess AT, Stoll V, Orchard E, Neubauer S, et al. Differential flow improvements after valve replacements in bicuspid aortic valve disease: a cardiovascular magnetic resonance assessment. J Cardiovasc Magn Reson 2018;20:10.

71. Della Corte A, Body SC, Booher AM, Schaefers HJ, Milewski RK, Michelena HI, et al. Surgical treatment of bicuspid aortic valve disease: knowledge gaps and research perspectives. J Thorac Cardiovasc Surg 2014;147:1749-1757.e1.

72. Knauth AL, Gauvreau K, Powell AJ, Landzberg MJ, Walsh EP, Lock JE, et al. Ventricular size and function assessed by cardiac MRI predict major adverse clinical outcomes late after tetralogy of Fallot repair. Heart 2008;94:211-216.

73. Geva T. Indications for pulmonary valve replacement in repaired tetralogy of Fallot: the quest continues. Circulation 2013;128:1855-1857.

74. Sridharan S, Derrick G, Deanfield J, Taylor AM. Assessment of differential branch pulmonary blood flow: a comparative study of phase contrast magnetic resonance imaging and radionuclide lung perfusion imaging. Heart 2006;92:963-968.

75. Lee N, Taylor MD, Hor KN, Banerjee RK. Non-invasive evaluation of energy loss in the pulmonary arteries using $4 \mathrm{D}$ phase contrast MR measurement: a proof of concept. Biomed Eng OnLine 2013;12:93.

76. Gewillig M. The Fontan circulation. Heart 2005;91:839-846.

77. Bächler P, Valverde I, Pinochet N, Nordmeyer S, Kuehne T, Crelier G, et al. Caval blood flow distribution in patients with Fontan circulation: quantification by using particle traces from $4 \mathrm{D}$ flow MR imaging. Radiology 2013;267:67-75.

78. Sjöberg P, Heiberg E, Wingren P, Ramgren Johansson J, Malm T, Arheden $\mathrm{H}$, et al. Decreased diastolic ventricular kinetic energy in young patients with Fontan circulation demonstrated by four-dimensional cardiac magnetic resonance imaging. Pediatr Cardiol 2017;38:669-680.

79. Taleb M, Khuder S, Tinkel J, Khouri SJ. The diagnostic accuracy of Doppler echocardiography in assessment of pulmonary artery systolic pressure: a meta-analysis. Echocardiography 2013;30:258-265.

80. Simonneau G, Gatzoulis MA, Adatia I, Celermajer D, Denton C, Ghofrani A, et al. Updated clinical classification of pulmonary hypertension. J Am Coll Cardiol 2013;62:D34-D41.

81. Boerrigter B, Mauritz G-J, Marcus JT, Helderman F, Postmus PE, Westerhof $\mathrm{N}$, et al. Progressive dilatation of the main pulmonary artery is a characteristic of pulmonary arterial hypertension and is not related to changes in pressure. Chest 2010;138:1395-1401.

82. Odagiri K, Inui N, Hakamata A, Inoue Y, Suda T, Takehara Y, et al. Noninvasive evaluation of pulmonary arterial blood flow and wall shear stress in pulmonary arterial hypertension with $3 \mathrm{D}$ phase contrast magnetic resonance imaging. SpringerPlus 2016;5:1071.

83. Fernandes T, Auger W, Fedullo P. Epidemiology and risk factors for chronic thromboembolic pulmonary hypertension. Thromb Res 2018 Jan 
8 [Epub]. https://doi.org/10.1016/j.thromres.2018.01.012.

84. Takagi H, Ota H, Sugimura K, Otani K, Tominaga J, Aoki T, et al. Dualenergy CT to estimate clinical severity of chronic thromboembolic pulmonary hypertension: comparison with invasive right heart catheterization. Eur J Radiol 2016;85:1574-1580.

85. Galiè N, Humbert M, Vachiery JL, Gibbs S, Lang I, Torbicki A, et al. 2015 ESC/ERS Guidelines for the diagnosis and treatment of pulmonary hypertension: The Joint Task Force for the Diagnosis and Treatment of Pulmonary Hypertension of the European Society of Cardiology (ESC) and the European Respiratory Society (ERS): Endorsed by: Association for European Paediatric and Congenital Cardiology (AEPC), International Society for Heart and Lung Transplantation (ISHLT). Eur Heart J 2016;37:67-119.

86. Guidelines for Treatment of Pulmonary Hypertension (JSC2012). http:// www.j-circ.or.jp/guideline/pdf/JCS2012_nakanishi_h.pdf/. Accessed November 11, 2017.

87. Uribe S, Beerbaum P, Sørensen TS, Rasmusson A, Razavi R, Schaeffter T. Four-dimensional (4D) flow of the whole heart and great vessels using real-time respiratory self-gating. Magn Reson Med 2009;62:984-992.

88. Frydrychowicz A, Winterer JT, Zaitsev M, Jung B, Hennig J, Langer M, et al. Visualization of iliac and proximal femoral artery hemodynamics using time-resolved 3D phase contrast MRI at 3T. J Magn Reson Imaging 2007;25:1085-1092. 